\title{
Case report of atypical scar endometriosis
}

\begin{abstract}
Scar endometriosis is an infrequent type of extra pelvic endometriosis that is rather close together with obstetrical and gynaecological surgeries. Scar endometriosis is reported in only $0.03-0.15 \%$ of all cases of endometriosis. We are reporting a case of 39 years old female patient presenting with scar endometriosis 10 years after her last lower segment caesarean section. The patient came to the Gynaec OPD, NIUM Hospital on $6^{\text {th }}$ May 2019 with the compliant of supra pubic swelling since 6months, which was growing slowly. Her menstrual history was regular, but she had lower abdominal pain during menstruation. Patient had two children delivered by LSCS. On clinical history, examination, USG and FNAC finding the swelling was diagnosed as scar endometriosis.
\end{abstract}

Keywords: endometriosis, abdominal scar, LSCS and prevention
Volume 7 Issue 5 - 2019

Tabassum K,Ambar S, Habiba S

Department of Obstetrics and Gynaecology, National Institute of Unani Medicine, India

Correspondence: Tabassum K, Associate professor, Dept of Obstetrics and Gynaecology, National Institute of Unani Medicine, Bangalore, India, Tel 7846074142 ,

Email drtabassum.nium@gmail.com

Received: July 22, 2019 | Published: September 30, 2019

\section{Introduction}

Endometriosis is defined as the presence of endometrial glands and stroma outside the uterus. It affects $10-15 \%$ of all women of reproductive age i.e. $18-45$ years, ${ }^{1,2}$ and $70 \%$ of women had chronic pelvic pain. ${ }^{3}$ Endometriosis occurs in abdominal, extra abdominal and remote areas. Scar endometriosis is reported in only $0.03-0.15 \%$ of all cases of endometriosis. Several reproductive factors have been consistently associated with risk for endometriosis, such as early age at menarche, ${ }^{4-6}$ late menopause, frequent menstrual cycles ${ }^{7,8}$ low parity, ${ }^{6,9,10}$ use of oestrogen pills, ${ }^{11}$ oestrogen producing tumours, obesity has also been put forward. ${ }^{12,13}$ The cause of endometriosis is unclear, but several theories have been reported. One possible mechanism is retrograde menstruation. This retrograde flow, along with potential hematogenous or lymphatic circulation, may result in the seeding of endometrial tissue in ectopic sites. Another theory is direct implantation of endometrial tissue during surgical procedures like LSCS, Hysterectomy, Myomectomy, Episiotomy etc. Other factors, such as genetic, environmental, hormonal, inflammatory or immunological may also result implantation of endometrial tissues on ectopic sites..$^{14-17}$

Clinical presentation of endometriosis varies in women. Patients often present with symptoms such as inter menstrual bleeding, dysmenorrhoea, dyspareunia, dyschezia and dysuria. ${ }^{18}$ Pelvic pain may present before onset of menstruation. Often, endometriosis can be asymptomatic, only diagnosed during evaluation for infertility. The lesions can be peritoneal lesions, superficial implants or cysts on the ovary, or deep infiltrating disease ${ }^{19}$ Classification of endometriosis associated symptoms have been established by the American Society for Reproductive Medicine (ASRM) based on the morphology of peritoneal and pelvic implants such as red or pink, white and black lesions, percentage of involvement of organ. Endometriosis in bowel, urinary tract, fallopian tube, vagina, cervix, skin or other locations are identified as per ASRM guidelines. Stages of endometriosis according to ASRM guidelines are stage I, II, III, and IV. These are based on the point scores and correspond to minimal, mild, moderate and severe endometriosis. Early diagnosis and intervention could ultimately improve the quality of life and preserve fertility. ${ }^{20}$

\section{Objective}

The goal of this paper is to highlight an atypical case of scar endometriosis.

\section{Case report}

A 39years old female patient came to Gynaec OPD of NIUM Hospital on $6^{\text {th }}$ May 2019 with the complaint of supra pubic swelling which was gradually increasing in size since last 6months. She also complained that the pain in lump is worse during menstruation. Her age of menarche was $15 y$ years. She is having regular menstruation with normal flow, but history of dysmenorrhoea is present. She had no history of other systemic illness. Her married life was 18years. She had two children both were delivered by LSCS and history of one spontaneous abortion was also present. The patient was tubectomized after caesarean section. Her last child birth was 10years back. According to her history before surgery she was fit and well with no documented history of endometriosis. Vitals were normal and her BMI was $26.2 \mathrm{Kg} / \mathrm{m}^{2}$.

On examination a supra pubic swelling size approximately $8 \times 5 \mathrm{~cm}$ was found at the site of lower part of caesarean scar. On palpation local temperature was raised, mass was irregular extending to pelvic region. (Figure 1) On vaginal examination uterus found anteverted, bulky, mobile and fornices free. On initial examination it was diagnosed as a lump. Consultant gynaecologist advised for USG and Cytology of lump. USG was done on 14/5/2019 findings suggested that large well defined hypoechohic solid lesion approximately $8.6 \times 6.5 \times 7.1 \mathrm{cms}$ noted in subcuteneous plane in mid line of lower anterior abdominal wall and supra pubic region suggestive of endometriosis. For confirmation FNAC was done on the same day i.e. 14/5/2019 which reported benign cellular stromal fragments of endometrium with occasional benign glands. No granuloma or malignancy was found. These findings suggested endometriosis. Extra abdominal endometriosis occurs at the time of surgical procedures like myomectomy, hysterectomy or LSCS etc. Here in this case previous history of two LSCS was present, which suggested the swelling may be due to direct implantation of endometrial tissue during caesarean section. This endometrial tissue grows every month in response to hormones especially oestrogen. Oestrogen helps in proliferation of 
endometrial tissue every month, hence the supra pubic swelling was increasing slowly.
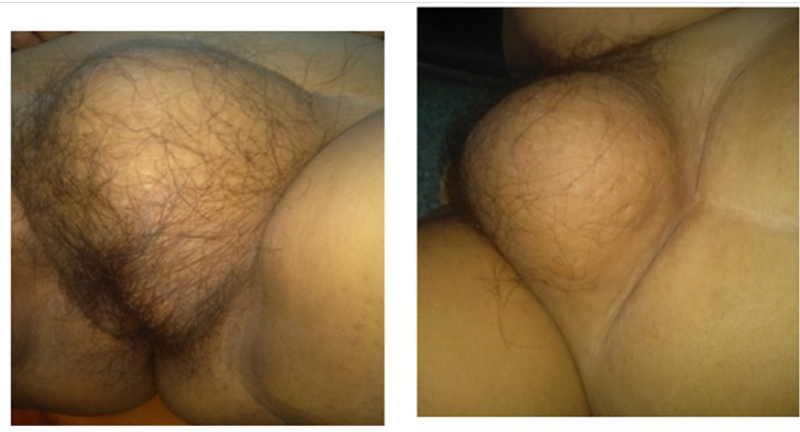

Figure I Pubic swelling.

\section{Discussion}

Scar endometriosis is rare entity usually follows previous abdominal surgery, especially early hysterectomy and caesarean section. There are numerous sites where extra pelvic endometriosis has been reported. These include lungs, pleura, bladder, kidney, bowel, omentum, umbilicus and abdominal wall. ${ }^{21}$ Endometriosis involving the abdominal wall is a rare occurrence, however, it should be considered in the differential diagnosis of abdominal wall masses in females. The typical clinical scenario involves a parous female with a history of gynaecological or obstetrician surgery presenting with a painful nodule or lump. The severity of pain and size of the lump may vary with menstrual cycle. In this case the patients remained without a diagnosis for 10years. Gynaecological history and physical examination is important in finalizing the diagnosis. The history revealed that the pain coincide with her menstrual cycle. The clinical examination revealed a soft, non reducible swelling located in pubic region. It was tender on palpation. On initial examination it was diagnosed as a lump. Based on the gynaecological history of a cyclical increase in the size and severity of pain an endometrioma was also included in the differential diagnosis. ${ }^{22}$

Surgical scar endometriosis is believed to result from deposits of endometrial cells during surgical intervention. These cells are then stimulated by oestrogen to produce endometriomas. Although relatively uncommon, it was well documented in clinical practice that scar endometriosis occur with different types of incisions where contact has possibly occurred with endometrial tissue. ${ }^{23}$ The endometriomas may develop 1-20years post operatively, example include caesarean section, laparoscopy, tubal ligation and hysterectomy. Of these caesarean section and hysterectomy are the most common. ${ }^{24}$ The incident after caesarean section is difficult to determine, but estimates range from $0.03 \%$ to $0.47 \% .{ }^{25}$ Minaghlia et al analyzed 30years of incisional endometriosis after caesarean section reported the incidence of scar endometriosis to be $0.08 \%{ }^{26}$ Frequency of scar endometriosis increase by number of caesarean section and laparoscopy performed in recent years. ${ }^{27}$ Other authors have reported an incidence of $0.2 \%$ in all caesarean sections performed. ${ }^{(28)}$ To make a definitive preoperative diagnosis of endometriosis is difficult. ${ }^{28}$

Positive histology confirms the diagnosis of endometriosis; negative histology does not exclude it, whether histology should be obtained if peritoneal disease alone is controversial. Visual inspection is usually adequate but histological confirmation of at least one lesion is ideal. Compared to laparoscopy, transvaginal ultrasound has no value in diagnosing peritoneal endometriosis, but it is a useful tool both to make and to exclude the diagnosis of an ovarian endometrioma. TVS may have a role in the diagnosis of disease involving the bladder or rectum. Medical imaging plays a role in locating the mass and ruling out hernia and other conditions, for example lipoma, abscess and suture granuloma. MRI remains the most useful imaging modality to exclude other pathology. ${ }^{28} \mathrm{In}$ this case the scar endometriosis was diagnosed by USG and was confirmed by FNAC of the lump.

\section{Conclusion}

Endometriosis is a debilitating disease that impacts the quality of life of adolescent and adult patients. Delayed diagnosis is common and may lead to a decline in reproductive potential and fertility. A semi or non-invasive diagnostic biomarker would be a useful tool to identify patients early in the disease process and thus improving outcomes, including less pain and better fertility. The occurrence of abdominal wall scar endometriosis after caesarean section has been a definite entity; steps to prevent this complication have not been explained. Literature recommends that through cleaning, irrigation with saline and closure of abdominal wound will prevent scar endometriosis.

\section{Acknowledgments}

None.

\section{Conflicts of interest}

The authors declare that there is no conflict of interest.

\section{Funding}

None.

\section{References}

1. Giudice LC, Kao LC. Endometriosis. Lancet. 2004;364(9447):1789-1799.

2. Noaham KE. World Endometriosis Research Foundation Global Study of Women's Health consortium. Impact of endometriosis on quality of life and work productivity: a multicenter study across ten countries. Fertil Steril. 2011;96(2):366-373.

3. Carter JE. Combined hysteroscopic and laparoscopic findings in patients with chronic pelvic pain. JAm Assoc Gynecol Laparosc. 1994;2(1):43-47.

4. Missmer SA, Hankinson SE, Spiegelman D, et al. Incidence of Laparoscopically Confirmed Endometriosis by Demographic, Anthropometric, and Lifestyle Factors. American Journal of Epidemiology. 2014;160(8):784-796.

5. Darrow SL. Menstrual Cycle Characteristics and the Risk of Endometriosis. Epidemiology. 1993; 4(2):135-142.

6. Missmer S, Hankinson S, Spiegelman D, et al. Reproductive history and endometriosis among premenopausal women. Obstet Gynecol. 2004;104:965-974.

7. Matalliotakis I, Cakmak H, Fragouli Y, et al. Epidemiological characteristics in women with and without endometriosis in the Yale series. Archives of Gynecology and Obstetrics. 2008;277(5):389-393.

8. Sangi-Haghpeykar H, Poindexter Ar. Epidemiology of endometriosis among parous women. Obstet Gynecol. 1995;85(6):983-992.

9. Candiani G, Danesino V, Gastaldi A, et al. Reproductive and menstrual factors and risk of peritoneal and ovarian endometriosis. Fertil Steril. 2009;56(2):230-234.

10. Peterson CM, Johnstone EB, Hammoud AO, et al. Risk factors associated with endometriosis: importance of study population for characterizing 
disease in the ENDO Study. American Journal of Obstetrics and Gynaecology. 2013;208(6):451.

11. Vercellini P, Eskenazi B, Consonni D, et al. Oral contraceptives and risk of endometriosis: a systematic review and meta-analysis. Hum Reprod Update. 2011;17(2):159-170.

12. Apter D, Reinilä M, Vihko R. Some endocrine characteristics of early menarche, a risk factor for breast cancer, are preserved into adulthood. International Journal of Cancer. 1989;44(5):783-787.

13. Nisolle M, Donnez J. Peritoneal endometriosis, ovarian endometriosis, and adenomyotic nodules of the rectovaginal septum are three different entities. Fertil Steril. 1997;68(4):585-596.

14. Farland LV, Shah DK, Kvaskoff M, et al. Epidemiological and Clinical Risk Factors for Endometriosis. In: D'Hooghe T, Editor. Biomarkers for Endometriosis. Springer Science; New York. 2015;5(6):233-234.

15. Anaf V, Simon P, El Nakadi I, et al. Hyperalgesia, nerve infiltration and nerve growth factor expression in deep adenomyotic nodules, peritoneal and ovarian endometriosis. Hum Reprod. 2002;17(7):1895-1900.

16. Wang G, Tokushige N, Markham R, et al. Rich innervations of deep infiltrating endometriosis. Hum Reprod. 2011;24(4):827-834.

17. Berkley KJ, Rapkin AJ, Papka RE. The pain of endometriosis. Science. 2005;308:1587-589.

18. Sinaii N, Plumb K, Cotton L, et al. Differences in characteristics among 1,000 women with endometriosis based on extent of disease. Fertil Steril. 2008;89(3):538-545.

19. Nisolle M, Donnez J. Peritoneal endometriosis, ovarian endometriosis, and adenomyotic nodules of the rectovaginal septum are three different entities. Fertil Steril. 1997;68(4):585-596.

20. American Society for Reproductive Medicine. Revised American Society for Reproductive Medicine classification of endometriosis. Fertil Steril. 2015;67(5):817-821.

21. Markham SM, Carpente SE, Rock JA. Extrapelvic endometriosis. Obstet Gynecol Clin north Am. 1989;16(1):193-219.

22. Brenner C, Wohlgemuth S. Scar Endometriosis. Surg Gynecol Obstet. 1990;170(6):538-540.

23. Koger KE, Shatney CH. Hodge K, et al. Surgical scar endometroima. Surg Gynaecol Obstet. 1993;177(3):244-246.

24. Bumpers HI, Butler KL, Best IM. Endometroima of the abdominal wall. Am J Obstet Gynecol. 2002;187(6):1709-1710.

25. Wolf Y, Haddad R, Werbin N, et al. Endometriosis in abdominal scars: a diagnostic pitfall. Am Surg. 1996;62(12):1042-1044.

26. Minaghlia S, Mishell R, Ballanrd CA. Incisional endometriosis after caesarean section: a case series. J of Reproductive Med for the Obstet and Gynaecol. 2007;52(7):630-634.

27. Aydin O. Scar endometriosis-a gynaecological pathology often presented to the general surgeon rather than the gynaecologist. Report of two cases. Langebeck's archives of surgery. 2007;392(1):105-109.

28. Khanmash MR, Omari AK, Gasaimeh GR, et al. Abdominal wall endometriosis. An overlooked diagnosis. Saudi Med J. $2003 ; 24(5): 523-525$. 